\title{
AnI open Blood pressure in different ethnic groups (BP-Eth): a mixed methods study
}

\author{
Sally Wood, ${ }^{1}$ Una Martin, ${ }^{2}$ Paramjit Gill, ${ }^{1}$ Sheila M Greenfield, ${ }^{1}$ M Sayeed Haque, ${ }^{1}$ \\ Jonathan Mant, ${ }^{3}$ Mohammed A Mohammed, ${ }^{1}$ Gurdip Heer, ${ }^{1}$ Amanpreet Johal, ${ }^{1}$ \\ Ramandeep Kaur, ${ }^{1}$ Claire Schwartz, ${ }^{1}$ Richard J McManus ${ }^{4}$
}

To cite: Wood S, Martin U, Gill $P$, et al. Blood pressure in different ethnic groups (BPEth): a mixed methods study. BMJ Open 2012;2: 0001598 . doi:10.1136/bmjopen-2012001598

\section{- Prepublication history for this paper are available online. To view these files please visit the journal online (http://dx.doi.org/10.1136/ bmjopen-2012-001598).}

Received 1 June 2012 Accepted 26 June 2012

This final article is available for use under the terms of the Creative Commons Attribution Non-Commercial 2.0 Licence; see http://bmjopen.bmj.com

For numbered affiliations see end of article

Correspondence to Dr Sally Wood; WoodSY@ adf.bham.ac.uk

\section{ABSTRACT}

Introduction: People of South Asian, AfricanCaribbean and Irish ethnicity are known to have worse cardiovascular outcomes than those from the white British group. While the reasons underpinning this are complex, the effect of hypertension is both significant and modifiable. In recent years, there has been increasing interest in and uptake of 'out-of-office' methods for blood pressure (BP) monitoring. However, guidance in this area has been largely based on research among the white population. This study aims to answer the following questions: (1) How often and in what ways does blood pressure (BP) monitoring occur and how does this differ between white and the above minority ethnic populations. (2) Are the thresholds for diagnosis of hypertension, and treatment targets in hypertension comparable for white British and minority ethnic populations using different measurement modalities: office blood pressure, ambulatory BP monitoring and home monitoring? (3) What preferences for BP measurement do people from white and minority ethnic populations have? Methods and analysis: A mixed methods approach will be used including the following: (1) A postal survey sent to 8000 hypertensive and not-known-tobe-hypertensive people from all four ethnic groups will determine current patterns of $\mathrm{BP}$ monitoring. (2) A validation study will compare BP measurement by ambulatory monitoring with office standard measurement, office research measurement and home monitoring in 200 people from each of the ethnic groups concerned. (3) Focus groups organised by ethnicity and gender will gather qualitative data regarding patient preferences for and experiences of $\mathrm{BP}$ measurement in each of the given modalities.

The data collected from these phases will be analysed appropriately in order to answer the above research questions.

Ethics and dissemination: Ethical approval has been gained from the Black Country Research Ethics Committee: Ref 09/H1202/114. The results of this work will be disseminated via journal publication and conference presentation.

\section{INTRODUCTION}

Cardiovascular outcomes for people of South Asian, African-Caribbean and white Irish origin living in the UK are worse than those

\section{ARTICLE SUMMARY}

Article focus

- The blood pressure in different ethnic groups (BP-Eth) study will provide important new evidence regarding the comparability of the thresholds for diagnosis of hypertension and monitoring in hypertension between white and minority ethnic groups using different modalities of measurement.

- It will also provide useful information about current patterns of and preferences for BP monitoring by ethnicity.

Strengths and limitations of this study

- The BP-Eth study is the first to directly compare BPs between ethnic groups using different modalities of measurement. The sample size of 800 for the validation study is sufficient to detect a $5 \mathrm{~mm} \mathrm{Hg}$ difference in mean BP by ethnic group between measurement modalities. Furthermore, the postal survey of 8000 people will provide robust evidence regarding current patterns of $\mathrm{BP}$ monitoring in different ethnic groups, while the focus groups will enable the factors determining patient preferences for different modalities of BP measurement to be better understood. The ethnic comparison aspect of the study may be limited if it is not possible to recruit a sufficient number of participants from each ethnic group under consideration to the various phases.

\section{Key messages}

- The BP-Eth study will consider the accuracy and acceptability of home, ABPM and clinic readings in minority ethnic populations in relation to the white British group. It will then assess whether current diagnostic thresholds and treatment targets for different modalities of measurement are appropriate in these ethnic groups.

for the white British group. ${ }^{1}{ }^{2}$ For example, South Asians have a $40-50 \%$ greater risk of mortality from coronary heart disease (CHD) compared with the general population ${ }^{34}$ with evidence that the poorest groups of Pakistanis and Bangladeshis have the highest death rates. $^{2}{ }^{5} \quad 6$ The mortality of migrant Caribbeans from CHD is lower than the 
national average but stroke deaths are higher (in women by $57 \%$, men $24 \%$ ), with hypertension (HT) being the major associated risk factor. ${ }^{2}$ Furthermore, data available suggest that the mortality of UK-born Caribbeans is little better than for those who have migrated from their homeland. ${ }^{7}$ Similarly, the Irish living in Britain experience higher mortality from both CHD (in women by $20 \%$, men $24 \%$ ) and strokes (in women by $23 \%$, men by $38 \%) .{ }^{8}$ Little is known about CHD and stroke mortality among UK-born Irish people, but one study reported an increase of $51 \%$ in cardiovascular mortality for men with Irish names living in Scotland. ${ }^{9}$

This increase in cardiovascular risk in ethnic groups is probably due to an interplay of complex factors including genetics, lifestyle (ie smoking habits, diet, barriers to healthcare) and deprivation. ${ }^{7}$ Hypertension remains a significant and potentially treatable risk factor in all ethnic groups. For example, in a Bangladeshi population with type 2 diabetes one study found a prevalence of $23.2 \%$ for systolic hypertension. ${ }^{10}$ There is also evidence that hypertension may go undetected and under treated in minority ethnic groups. Cappuccio et al found a twofold to threefold increase in hypertension in South Asians and Caribbeans; only $49 \%$ of those with hypertension had adequate control; $18 \%$ were undiagnosed before the survey and $17 \%$ were not receiving medication. ${ }^{11}$

\section{Blood pressure monitoring}

Increased availability of various automated devices has encouraged individuals to monitor their blood pressure at home. The use of ambulatory blood pressure monitoring (ABPM) has also led to a realisation that multiple readings may improve accuracy of diagnosis. In general, both ABPM and home monitoring may help to improve treatment, ${ }^{12}{ }^{13}$ identify resistant $\mathrm{HT}^{14}{ }^{14}$ diagnose white coat $\mathrm{HT}^{15-17}$ and predict cardiovascular outcomes. ${ }^{18-20}$ ABPM is the only method that can identify reduced night time dipping which is a poor prognostic indicator. $^{21}$ The definitive diagnosis of white-coat HT by means of ABPM may ultimately improve health outcomes and reduce healthcare costs. ${ }^{20}$

Few studies of blood pressure monitoring undertaken over the last 20 years have included people from South Asian, African-Caribbean or white Irish populations with the result that very little is known about comparative measurements including self-monitoring. For instance, it is not clear as to whether the 'white coat' effect seen in white British populations is similar, greater or less among these minority ethnic communities. Nor is it known whether observed differences between office and home measurements among the white group are similar or different in South Asian or African-Caribbean populations.

\section{Diagnosis and management of blood pressure}

The diagnosis and management of blood pressure are informed by guidelines largely based on research from white populations. ${ }^{10} 22$ These guidelines recommend diagnostic and treatment thresholds for hypertension on the basis of office blood pressure and $24 \mathrm{~h} \mathrm{ABPM}$ or home blood pressure monitoring. Indeed, the recent NICE guidance for the management of hypertension ${ }^{10}$ uses factors to adjust between clinic and 'out-of-office' thresholds for diagnosis that were derived from Australian data gathered in a population that was $82 \%$ white and $15 \%$ Asian. ${ }^{23}$ These factors are a decrease of $5 / 5 \mathrm{~mm} \mathrm{Hg}$ when converting from clinic to out-of-office measured blood pressures at lower levels (stage 1 threshold) and a corresponding decrease of $10 / 5 \mathrm{~mm} \mathrm{Hg}$ at higher levels (stage 2 threshold). At present, ethnicity is not considered in the specification of these thresholds, treatment targets or adjustment factors.

\section{Purpose}

The BP-Eth study will consider the accuracy and acceptability of home, ABPM and clinic readings in minority ethnic populations in relation to the white British group. It will then assess whether current diagnostic thresholds and treatment targets for different modalities of measurement are appropriate in these ethnic groups.

\section{METHODS}

\section{Overview of methods}

BP-Eth is a primary care-based mixed methods observational study involving both quantitative and qualitative elements.

Study has three phases

Phase 1-postal cross-sectional survey

Phase 2-validation study

Phase 3-focus group study

\section{Population}

The study population will comprise people both with and without diagnosed hypertension recruited from primary care. Eligibility criteria will be aged between 40 and 74 years and belonging to one of the four ethnic groups under investigation (white British, white Irish, South Asian, African-Caribbean). Patients who are unable to consent to participation belong to a different ethnic group or who's general practitioner feels they are unable to take part will be excluded. Participants will need to have had at least one blood pressure recorded in their electronic medical records within the last 5 years.

\section{Setting}

Patients will be identified from practices who are members of the Central England Primary Care Research Network (PCRN-CE). This includes around 300 practices in the West Midlands which have been shown to be generalisable to wider primary care. ${ }^{24}$ Approximately 20 practices will be recruited to participate in this study. 
Phase 1 postal cross-sectional survey

Procedures

A cross-sectional survey of 8000 people including representative samples of both HT and not known to be hypertensive (NHT) individuals from the four ethnic groups under consideration will elucidate current blood pressure monitoring patterns (self, third party, eg, pharmacy, etc, health professional), confirm ethnic group and identify participants for the validation study. This postal questionnaire will be sent to approximately 4000 people with a Read Code of hypertension in their electronic medical notes and 4000 with no such Read Code. A list of eligible participants from each practice will be generated from the criteria specified above. An equal number of participants with and without hypertension will be randomly selected to receive the survey. Practices will be chosen from areas likely to include appropriate populations based on ward-level census data and personal knowledge of the investigators. The survey questionnaire will be accompanied by a covering letter translated into the relevant languages, with telephone follow-up of non-responders by a bilingual researcher. Responses will be entered into a secure database.

\section{Outcome measures}

The primary outcome measure will be prevalence of self, professional (practice, pharmacy and outpatient) and ambulatory monitoring over the last 12 months in each ethnic group. Secondary outcomes will include preferences for different types of monitoring.

\section{Sample size considerations}

Phase 1 questionnaires will be sent to a random sample of 8000 people (see above). A 50\% response rate (realistic in this population from previous work) would result in 4000 responses. It is anticipated that a proportion of responses will fall outside of the four ethnic groups being studied; hence further questionnaires will be sent as required (up to 10000 ) in order to receive responses from 1000 individuals in each ethnic group under consideration, half of which will have hypertension and half will not . This will allow estimation of the overall prevalence of the different types of monitoring with and without hypertension to within $2.7 \%$ assuming a $10 \%$ prevalence of monitoring in each case (the approximate community prevalence of self-monitoring in a white population). ${ }^{25}$

\section{Analysis}

The overall prevalence of blood pressure monitoring will first be estimated. Thereafter, the variation in its prevalence by ethnic group, age, sex, employment status and deprivation will be explored using logistic regression models which may also incorporate a random effects term for general practices.
Phase 2 validation study

\section{Procedures}

Phase 2 is a validation study comparing blood pressure monitored in a clinic setting with ambulatory and home measurements. Participants in this phase will be asked to measure their blood pressure using all three of these methods. Recruitment will be from those responding to phase 1 who indicate a willingness to participate in phase 2. Such individuals will then be invited to attend clinics run at their own practices. Blood pressure measurements and study questionnaires will be undertaken along with training regarding both ambulatory and home monitoring. Figure 1 shows how patients move through the various different methods included in this phase while table 1 shows the data that will be collected as a result. The order of out-of-office blood pressure measurement (home or ambulatory) will be randomised so that approximately equal numbers of individuals will have home or ambulatory first. People with and without hypertension will be invited to undertake phase 2 so that approximately equal proportions of each are included. Upon completion of this phase, participants will be asked whether they would be willing to take part in phase 3 .

\section{Outcome measures}

The primary outcome will be the mean difference between the reference standard (mean daytime ambulatory blood pressure) and standard office (mean of second and third readings on three occasions), mean home monitored BP (last 24 readings), office research (mean of second to sixth readings on three occasions) and the last routine practice blood pressure recorded in the clinical records. Each different ethnic group will be considered separately with subgroups of those treated for hypertension and not receiving treatment. The effect of these differences on standard diagnostic and treatment target thresholds will be evaluated (ie 140/ $90 \mathrm{~mm} \mathrm{Hg}$ for clinic readings and 135/85 $\mathrm{mm} \mathrm{Hg}$ for out of office measurement at the stage 1 threshold and the equivalents at the stage 2 threshold). ${ }^{11}$

\section{Sample size considerations}

About 100 patients with and without hypertension will be recruited from each ethnic group. On the basis of previous work in a white population, 200 patients per ethnic group, that is, 800 people in total, will be sufficient to detect a difference of $5 \mathrm{~mm} \mathrm{Hg}$ in mean differences between any two populations (this is sufficient across the plausible range of SDs between 12 and $18 \mathrm{~mm} \mathrm{Hg}$, power 80\%). Differences of less than $5 \mathrm{~mm} \mathrm{Hg}$ are unlikely to be clinically significant given the day-to-day variation of blood pressure within individuals. A further $5 \%$ approximately will be recruited as required to account for drop-outs or equipment malfunction. 


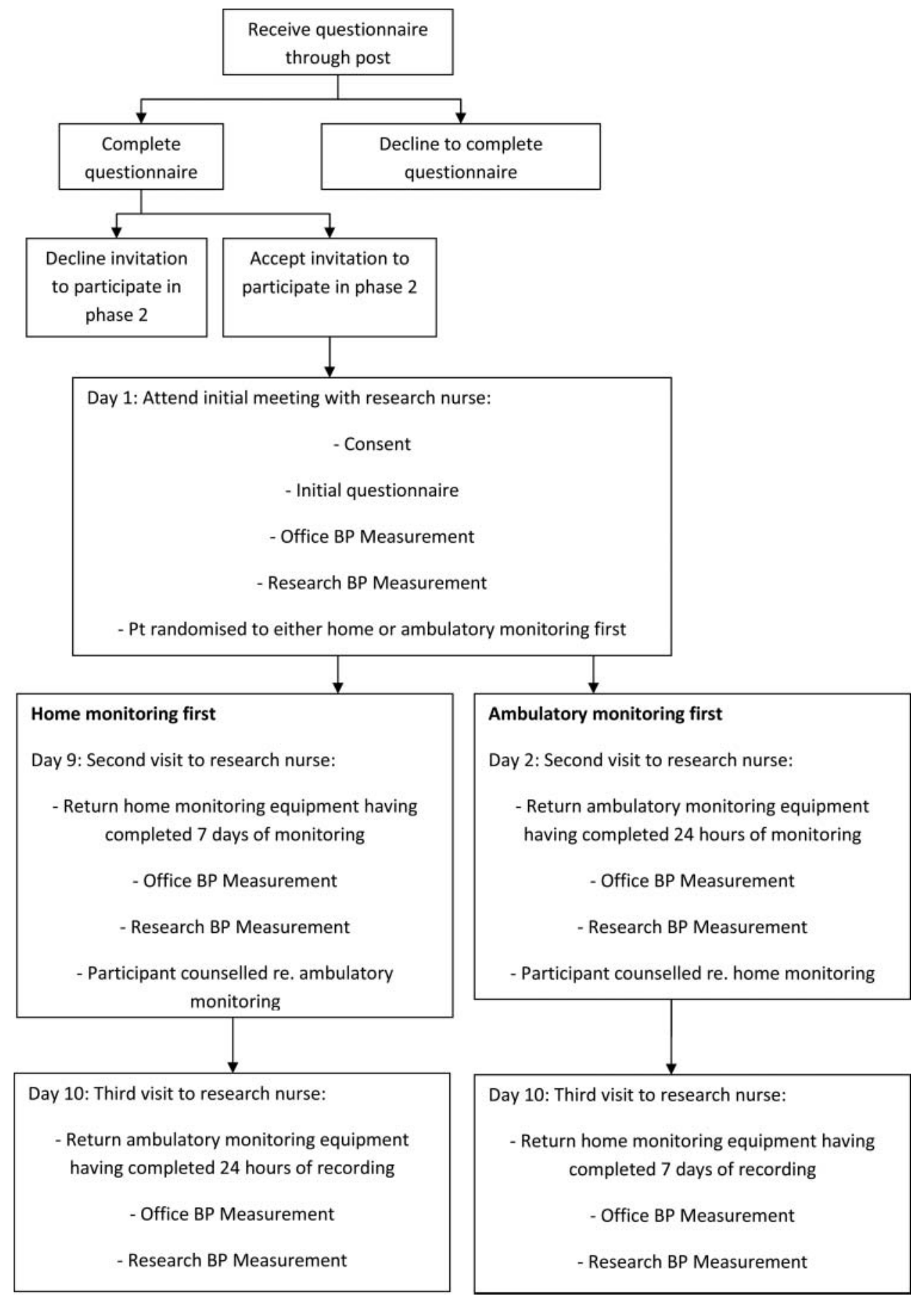

Figure 1 Patient flow through phase 2.

\section{Analysis}

Between groups, $\mathrm{t}$ tests will be used to compare mean differences in ambulatory versus office, home-monitored and routine blood pressures between white British, South Asian, African-Caribbean, white Irish populations separately for people with a diagnosis of hypertension, and for people without a prior diagnosis of hypertension (ambulatory used as reference standard). Since we are interested in the differences between each minority ethnic group and the white British group, each comparison is of interest and will be dealt with individually. Thus, no adjustment for multiple comparisons is required. Within groups, repeated measures general linear modelling (GLM) and mixed effects models will be used to evaluate differences between the different methods of measurement and routinely collected BP data with post hoc tests where significant differences are found. Baseline covariates will be examined for similar age/gender/blood pressure distribution and adjustment will be incorporated in the analysis where necessary. 


\section{Table 1 Summary of data collected during phase 2

\begin{tabular}{ll}
\hline Questionnaires & Demographic details \\
\hline Medical history \\
Antihypertensive and other relevant medication \\
Smoking status and alcohol consumption \\
Ethnicity \\
Place of birth \\
Years residence in UK \\
Spoken languages (first and any others) \\
Religion \\
Marital status and highest educational qualification \\
Beliefs about medicines questionnaire-as per that used by Home et a $\beta^{32}$ \\
Blood pressure monitoring acceptability questionnaire (for each of the three types of monitoring) \\
- as per that used by Little et a $\beta^{33}$ \\
Blood pressure monitoring preference questionnaire
\end{tabular}

Physical measurements Height

Weight

Waist circumference

Blood pressure measurements

Clinic blood pressure using BP-Tru Sphygmomanometer measured on three occasions with bilateral simultaneous measurement on the first occasion

Ambulatory blood pressure measurement over $24 \mathrm{~h}$ with half hourly measurement 8:00-23:00 and hourly measurement 23:00-8:00

Home blood pressure measurement, two readings twice daily for 7 days, that is, 28 readings total
Differences will be investigated to assess any relationship to the level of blood pressure. A significance level of $\mathrm{p}<0.05$ will be used and sensitivity analysis will examine the potential effect of missing data. Analyses will be performed at the end of the study after all data have been collected. No interim analysis will be performed as this is an observational study. Planned subgroup analyses will be undertaken for diabetic versus non-diabetic patients, older versus younger (65 as threshold), males versus females, higher versus lower blood pressure (threshold 150 systolic).

\section{Phase 3 focus group study}

\section{Procedures}

Focus groups comprising participants who have completed phases 1 and 2 will consider patient preferences for and experiences of blood pressure measurement in each of the three ways included in the study. Eight groups will be organised according to gender and ethnicity. The former is necessary in order to achieve the research objectives. Meanwhile, it is well known that males and females may interact differently in mixed-gender as opposed to same-gender groups. ${ }^{26}$ It is therefore anticipated that organising by gender will enabled a more liberal exchange of views across all ethnicities. It is also hoped that each group will comprise an adequate mix of HT and NHT patients. However, given the difficulties of gathering the requisite number of participants (see below) with the same gender and ethnicity at a given time and location, recruitment will be independent of hypertensive status. While each group will be held at a participating practice, participants may be drawn from many different practices: however, they will all have finished phase 2 within the last 6 months (any longer than this may result in recall issues). Each group will be facilitated by a researcher according to a topic guide which will comprise a predetermined set of questions developed by the study steering group. Each session will be recorded, while a co-researcher will also attend to make a note of the opening words used by each participant in order to enable identification later on. Interviews will be transcribed verbatim for analysis. It is envisaged that each focus group will be conducted in English. However, if it emerges that the South Asian participants agreeing to attend the group would like to converse in an alternative language then this will be arranged through the recruitment of a facilitator with the appropriate linguistic skills, and subsequent translation of the corresponding transcript back into English.

\section{Outcome measures}

This phase of the study will explore preferences for and acceptability of different modalities of blood pressure measurement by ethnic group.

\section{Sample size considerations}

Eight focus groups will be organised, as mentioned above, to ethnicity and gender. Research suggests an optimal focus group size of between 5 and 10 
participants. ${ }^{27}$ The proposed group size here is between 6 and 8 individuals in order to capture a variety of views in response to each question on the topic guide within a $1.5 \mathrm{~h}$ time frame. Given likely attrition rates of around $20 \%,{ }^{28} 10$ participants will be recruited to each of the focus groups in order to achieve the target size.

\section{Analysis}

A 'thematic' approach will be used in the analysis of the focus group transcripts. This is ideally suited to identifying the ideas and relationships that underpin preferences for each modality of $\mathrm{BP}^{29}$ Here, textual data in transcripts will be grouped into meaningful categories ('themes') in order to represent a range of attitudes and ideas along with otherwise unarticulated social values. ${ }^{30}$ As new transcripts are produced for later focus groups the themes may be revised. Each coded transcript will then be passed to a second researcher for triangulation purposes. The purpose of the analysis is to compare themes within, between and across ethnicities: a matrix will be constructed in order to facilitate this. Patterns and trends will then be identified and their basis will be considered. Where relevant, the interaction between participants will be analysed in order to ascertain how knowledge is constructed within the group setting. Here, an analytical template proposed by Lehoux $e t a l^{81}$ will be used.

\section{Recruitment}

Twenty practices with mean list sizes of 5000 adult patients (lower than usual list sizes to reflect the typical practice sizes seen in majority ethnic population areas such as the inner city) and a conservative prevalence of hypertension of $10 \%$ will result in a potential sample of at least 10000 patients with hypertension and many times this number without. This will be sufficient for the invitations needed for phase 1 and respondents will subsequently be recruited into phases 2 and 3 . Further practices may be required later on to ensure an adequate mix of ethnicities.

\section{Staff training}

All staff involved in the study will undergo training given by the lead research nurse in order to ensure a consistent approach. Work instructions detailing the procedures to be followed in each of the different phases will be made available. These will describe the action to be taken in the instance of unusually high or low readings, a significant inter-arm difference and severe bruising/ allergy from use of a blood pressure cuff.

\section{Ethics and dissemination}

Ethical approval has been gained from the Black Country Research Ethics Committee: Ref 09/H1202/ 114. The results of this work will be disseminated via journal publication, conference presentation and feedback to participating practices.

\section{DISCUSSION}

The results of this study will be relevant to UK primary care as information about norms and preferences for ambulatory and self-monitoring in minority ethnic groups is vital to allow optimum care to be provided both in the diagnosis and in the management of hypertension. Determining the relationship between home/ ambulatory blood pressure monitoring and standard office readings in each ethnic group will allow consideration to be made of whether the current thresholds for diagnosis of hypertension, and treatment targets in hypertension, are universally appropriate. Furthermore, it will also enable adjustment factors between different methods of blood pressure measurement to be derived for each ethnic group.

\section{Author affiliations}

${ }^{1}$ Primary Care Clinical Sciences, NIHR School for Primary Care Research, University of Birmingham, Edgbaston, Birmingham, UK

${ }^{2}$ Clinical Pharmacology, University of Birmingham, Edgbaston,

Birmingham, UK

${ }^{3}$ Primary Care Unit, University of Cambridge, Cambridge, UK

${ }^{4}$ Primary Care Health Sciences, NIHR School for Primary Care Research, University of Oxford, Oxford, UK

Acknowledgements Mr Roger Holder, Head of Statistics at Primary Care Clinical Sciences, University of Birmingham and Dr Jamie Coleman, Consultant Clinical Pharmacologist at University Hospital Birmingham were original coapplicants who assisted in the design of this study before moving on to other projects. The authors would like to acknowledge their contribution to this work.

Contributors RJM and UM had the original idea for this work and gained funding in collaboration with PG, JM, SG, JC and MM. SW wrote the first draft of this paper and all authors subsequently assisted in redrafting and have approved the final version. RJM will act as guarantor.

\section{Competing interests None.}

Funding NIHR Research for Patient Benefit; Service support costs via Birmingham and the Black Country Comprehensive Local Research Network, recruitment supported by the Central England Primary Care Research Network (PCRN-CE). RJM is supported by an NIHR Career Development Fellowship.

Both Primary Care Clinical Sciences, University of Birmingham and Primary Care Health Sciences, University of Oxford, are members of the NIHR School for Primary Care Research.

Provenance and peer review Not commissioned; internally peer reviewed.

\section{REFERENCES}

1. Gill PS, Kai J, Bhopal RS, et al. Health Care Needs Assessment: Black and Minority Ethnic Groups. (book auth.) Stevens A, Mant J Rafferty J. Health Care Needs Assessment. The epidemiologically based needs assessment reviews. Third series. Abingdon: Radcliffe Publishing Ltd, 2007.

2. Capuccio FP, Kerry S, Forbes L, et al. Blood pressure control by home monitoring: meta-analysis of randomised trials. BMJ 2004;329:145

3. Balarajan R. Ethnicity and variations in mortality from coronary heart disease. Health Trends 1996;29:45-51.

4. Bhopal R, Sengupta-Weibe S. Cardiovascular risks and outcomes: ethnic variations in hypertensive patients. Heart 2000;83:495-6.

5. Bhopal R, Unwin N, White M, et al. Heterogeneity of coronary heart disease risk factors in Indian, Pakistani, Bangladeshi and European origin populations: cross sectional study. BMJ 1999;319:215-20.

6. Nazroo J. The health of Britain's ethnic minorities. London: Policy Studies Institute, 1997. 
7. Abbotts J. Cardiovascular risk profiles in UK-born Caribbeans and Irish living in England and Wales. Atherosclerosis 2004;175:295-303.

8. Wild S, McKeigue P. Cross sectional analysis of mortality by country of birth in England and Wales. BMJ 1997;314:705-10.

9. Abbotts J, Williams R, Smith G. Association of medical, physiological, behavioural and socio-economic factors with elevated mortality in men or Irish heritage in West Scotland. J Public Health Med 1999;21:46-54.

10. (NICE) National Institute for Clinical Excellence. CG127 Hypertension - the clinical management of primary hypertension in adults. 2011. http://www.nice.org.uk/nicemedia/live/13561/56007/ 56007.pdfcv

11. Cappucio FP, Cook D, Atkinson R, et al. Prevalence, detection and management of cardiovascular risk factors in different ethnic groups in south Longon. Heart 1997;78:555-63.

12. Cappucio FP, Kerry SM, Forbes L. Blood pressure control by home monitoring: meta-analysis of randomised trials. BMJ 2004;329:145.

13. Pickering T, O'Brien E. Second international consensus meeting on 24-hour ambulatory BP measurement: consensus and conclusions. J. Hypertens 1991;9:563-5.

14. O'Brien E, Coats $\mathrm{A}$, Owens $\mathrm{P}$, et al. Use and interpretation of ambulatory blood pressure monitoring: recommendations of the British Hypertension Society. BMJ 2000;320:1128-34.

15. European Society of Hypertension. European Society of Cardiology guidelines for the management of arterial hypertension. J Hypertens 2003;21:1011-53.

16. Appel L, Stason WB. Ambulatory blood pressure monitoring and blood pressure self-measurement in the diagnosis and management of hypertension. Ann Intern Med 1993;118:867-82.

17. Chobanian AV, Bakris GL, Black HR, et al. Seventh report of the joint national committee on prevention, detection, evaluation and treatment of high blood pressure. Hypertension 2003;42:1206-52.

18. Imai $\mathrm{Y}$, Ohkubo T, Sakuma M, et al. Predictive power of screening blood pressure, ambulatory blood pressure and blood pressure measured at home for overall and cardiovascular mortality: a prospective observation in a cohort from Ohasama northern Japan. Blood Press Monit 1996;1:251-4.

19. Ohkubo T, Hozawa A, Nagai K, et al. Prediction of stroke by ambulatory blood pressure monitoring versus screening blood pressure measurements in a general population: the Ohasama study. J Hypertens 2003;21:821-48.
20. O'Brien E, Asmar R, Beilin L, et al. European Society of Hypertension recommendations for conventional, ambulatory and home blood pressure measurement. J Hypertens 2003;21:821-48.

21. Ohkubo T, Hozawa A, Yamaguchi J, et al. Prognostic signficance of the nocturnal decline in blood pressure in individuals with and without high $24 \mathrm{~h}$ blood pressure: the Ohasama study. $J$ Hypertens 2002;20:2183-9.

22. Williams B, Poulter N, Brown M, et al. British Hypertension Society guidelines for hypertension management 2004 (BHS-IV): summary. BMJ 328, 2004;7440:634-40.

23. Head GA, Mihailidou A, Duggan K, et al. Definition of ambulatory blood pressure targets for diagnosis and treatment of hypertension in relation to clinic blood pressure: a prospective cohort study. BMJ 2010;340:1104.

24. McManus RJ, Ryan R, Jones $M$, et al. How representative of primary care are research active practices? Cross-sectional survey. Family Pract 2008;25:56-62.

25. McManus RJ, Ryan A, Greenfield S, et al. Self measurement of blood pressure: a community survey. J Hum Hypertens 2007;21:741-3.

26. Stewart DW, Shamdasani PN. Focus groups: theory and practice. Thousand Oaks, CA: Sage Publications, 1990.

27. Osborn A. Applied imagination: principles and procedures of creating thinking. New York: Charles Scribner's Sons, 1953.

28. Robinson N. The use of focus group methodology-with selected examples from sexual health research. J Adv Nurs 29, 1999;4:905-13.

29. Kruegar R, Casey M. Focus groups: a practical guide for applied research. 3rd edn. Thousand Oaks, CA: Sage Publications, 2000.

30. Kamberelis G, Dimitriadis G. Focus Groups: Strategic articulations of pedagogy, politics and inquiry. (book auth.) Danzin NK, Lincoln YS, eds. Collecting and interpreting qualitative materals. 3rd edn. Thousand Oaks, CA: Sage Publications, 2008:375-402.

31. Lehoux P, Poland B, Daudeline G. Focus group research and 'the patient's view'. Soc Sci Med 2006;63:2091-104.

32. Home R, Weinman J, Hankins M. The beliefs about medicines questionnaire: the development and evaluation of a new method for assessing the cognitive representation of medication. Psychol Health 1999;14:1-24.

33. Little P, Barnett J, Barnsley L, et al. Comparison of the acceptability of and preferences for different methods of measuring blood pressure in primary care. BMJ 325, 2002;7358:258-9. 